\title{
Effects of the $\beta$-Adrenoceptor Blocker Carvedilol in Short QT Syndrome Caused by N588K Mutation in HERG: A Simulation Study
}

\author{
Cunjin Luo ${ }^{1,2}$, Linghua $\mathrm{Li}^{3}$, Tong $\mathrm{Liu}^{4}$, Kuanquan $\mathrm{Wang}^{2}$, Xiangyun $\mathrm{Bai}^{2}$, Ying $\mathrm{He}^{5}$, Henggui \\ Zhang ${ }^{1,2,6}$ \\ ${ }^{1}$ Key Laboratory of Medical Electrophysiology, Ministry of Education, Institute of Cardiovascular \\ Research, Southwest Medical University, Luzhou, China \\ ${ }^{2}$ School of Computer Science and Technology, Harbin Institute of Technology, Harbin, China \\ ${ }^{3}$ School of Integrative Medicine, Southwest Medical University, Luzhou, China \\ ${ }^{4}$ Key Laboratory of Ionic-Molecular Function of Cardiovascular Disease, Tianjin Institute of \\ Cardiology, Second Hospital of Tianjin Medical University, Tianjin, China \\ ${ }^{5}$ School of Computer Science and Informatics, De Montfort University, Leicester, UK \\ ${ }^{6}$ School of Physics and Astronomy, The University of Manchester, Manchester, UK
}

\begin{abstract}
The short QT syndrome (SQTS) is associated with shortening of $Q T$ interval resulting from an accelerated cardiac repolarization. The SQT1, SQTS variant, results from a gain-of-function N588K-KCNH2 mutation in the rapid delayed rectifier potassium current $\left(I_{K r}\right)$ channels. Since $\beta$-Adrenoceptor blocker can block slow delayed rectifier potassium currents $\left(I_{K s}\right)$ and $I_{K r}$, we used in silico approach to evaluate carvedilol's effects on SQT1.

Mathematical models of human ventricular action potential (AP) developed by ten Tusscher et al. were modified to incorporate a Markov chain formulation of $I_{K r}$ describing the SQT1 mutant condition. AP models were incorporated into a transmural strand for investigation of $Q T$ interval changes. In addition, the simulated $I_{K s}$ and $I_{K r}$ inhibition to prolong the QT interval in SQT1 was quantified. The blocking effects of carvedilol on $I_{K s}$ and $I_{K r}$ were modelled by using Hill coefficient and $I C_{50}$ from literatures $\left(10 \mu M\right.$ carvedilol reduced $I_{K r}$ in Wild Type- and N588K-KCNH2 by $92.8 \%$ and $36.0 \%$; it reduced $I_{K s}$ by $36.5 \%$ in both conditions). At single cell level, carvedilol prolonged the AP duration (APD) in SQT1; at strand level, the effects of carvedilol normalized the QT interval in SQT1 from $286 \mathrm{~ms}$ to $364 \mathrm{~ms}$.

Simulations identified $\beta$-Adrenoceptor blocker carvedilol as a potential drug for SQTS treatment.
\end{abstract}

\section{Introduction}

The short QT syndrome (SQTS) is a cardiac disorder associated with abnormally short QT interval on the ECG, leading to increased risk of atrial and/or ventricular arrhythmias and sudden cardiac death [1,2]. The SQTS is genetically heterogeneous, with a complex genotypephenotype relationship. The first identified form of the SQTS (SQT1) [3] was caused by a missense mutation (N588K) to the human $h E R G$ encoding the channels carrying the rapid delayed rectifier potassium current, $I_{\mathrm{Kr}}$. Studies [2,4] showed the N588K- $h E R G$ mutation significantly attenuated inactivation, without altering the voltage dependence of activation, causing a "gain-offunction" in $I_{\mathrm{Kr}}$ which significantly reduces the QT interval (QTc $\leq 300 \mathrm{~ms})$.

The current first-line treatment for SQTS patients is use of an implantable cardioverter-defibrillator (ICD) device, which protects against SCD [5]. However, Twave over-sensing, which leads to erroneous identification of tachyarrhythmic events, can be an issue with such devices, as T-waves often appear tall and peaked in SQTS patients. Furthermore, ICD does not restore the QT interval and, is not suited to some pediatric patients, necessitating the pursuance of alternative, pharmacological approaches. Pharmacological therapy may be the primary modality to restore the physiological (normal) QT interval and protect against arrhythmias. At present, the accurate experimental models of SQT1 and in vitro pharmacological data on SQT1 patients are comparatively sparse [2,4]. However, previous studies have reported that $\beta$-adrenoceptor blocker carvedilol has some efficacy in reversing the SQTS phenotype [6]. Detailed in vitro studies into the pharmacology of N588K-KCNH2 and V307L-KCNQ1 mutations used patch clamp measurements to assess the blocking potency of $\beta$-adrenoceptor blocker carvedilol on $I_{\mathrm{Ks}}$ and $I_{\mathrm{Kr}}$ currents [6]. In this study, $\beta$-adrenoceptor blocker carvedilol emerged as a potential agent for treating SQTS.

The underlying mechanisms by which combined ion 
channel blocking actions of carvedilol exert antiarrhythmic effects in the setting of SQT1 are not known. Whereas, several studies [1,2,7-10] have previously used computer models to gain insights into QT interval shortening and pro-arrhythmic effects of SQT1 mutant $h E R G$ channels in human ventricles, significantly less is known about the pharmacological agents on human ventricular electrophysiology in SQT1 variant. Our simulation studies adopted a simplified "pore block" approach to investigate the effects of drugs on SQTS $[2,10]$. The present study was also adopted simplified "pore block" approach to provide mechanistic information regarding the actions of carvedilol on human ventricular electrophysiology in the setting of N588Klinked SQT1.

\section{Methods}

\subsection{Model development}

The ten Tusscher model [11,12] of the human ventricular action potential (AP) was used for simulations in this study, due to its extensive experimental validation and ability to reproduce complex behaviours when simulating anti-arrhythmic effects of pharmacological agents on cardiac electrophysiology. An updated form of the ten Tusscher model described by Zhang et al. [4] was used, as this configuration gave a QT interval shortening which was more concordant with clinical observed in the SQTS.

Specifically, the single cell model can be modelled by using the following ordinary differential equation (ODE):

$$
\frac{d V_{m}}{d t}=-\frac{I_{\text {ion }}+I_{s t i m}}{C_{m}}
$$

where $t$ is time, $C_{\mathrm{m}}$ is the cell membrane capacitance, $I_{\mathrm{stim}}$ is the external stimulus current and $I_{\text {ion }}$ is the sum of the transmembrane currents. The late sodium current $\left(I_{\mathrm{NaL}}\right)$ equation [13] was incorporated. The model code used in this study was downloaded from http://wwwbinf.bio.uu.nl/khwjtuss/. The cell model was paced with an amplitude of $-52 \mathrm{pA} / \mathrm{pF}$ for $1 \mathrm{~ms}$ and a basic cycle length (BCL) of $800 \mathrm{~ms}$.

To simulate the electrophysiological effects of the $K C N H 2 \mathrm{~N} 588 \mathrm{~K}$ mutation, the parameters of $I_{\mathrm{Kr}}$ equations were modified to incorporate the experimentally-observed kinetic properties of $I_{\mathrm{Kr}}$ channels [4]. As SQTS mutations are expressed heterozygously in vivo, and according to our previous work [4], the modified parameters of the $I_{\mathrm{Kr}}$ equations are presented below.

Original:

$$
I_{K r}=G_{K r} \times O_{K r} \times\left(V_{m}-E_{K r}\right)
$$

$$
\begin{gathered}
G_{K r}=0.0243 \times\left[K^{+}\right]_{o}^{0.59} n S / p F \\
E_{K r}=\frac{R T}{F} \log \frac{\left[K^{+}\right]_{o}}{\left[K^{+}\right]_{i}}
\end{gathered}
$$

WT:

$$
\begin{aligned}
& \alpha_{1}=2.172 \\
& \beta_{1}=1.077
\end{aligned}
$$

$$
\alpha_{2}=0.00655 \times e^{0.027735765 \times(V-36)}
$$

$$
\beta_{2}=0.001908205 \times e^{0.0148902 V}
$$

$$
\begin{gathered}
\alpha_{i}=0.04829 \times e^{-0.039984 \times(V+25)} \times\left(\frac{4.5}{K_{o}}\right) \\
\beta_{i}=0.2624 \times e^{0.000942 V} \times\left(\frac{4.5}{K_{o}}\right)^{0.3} \\
\alpha=0.00555 \times e^{0.05547153 \times(V-12)} \\
\beta=0.002357 \times e^{-0.036588 V} \\
\mu=\frac{\alpha_{i} \beta_{2}}{\beta_{i}}
\end{gathered}
$$

N588K:

$$
\begin{array}{r}
\alpha_{1}=2.172 \\
\beta_{1}=0.5385
\end{array}
$$

$$
\alpha_{2}=0.001965 \times e^{0.05547153 \times(V-21)}
$$

$$
\beta_{2}=2.260489 \times 10^{-6} \times e^{-0.0925782 V}
$$




$$
\begin{gathered}
\alpha_{i}=0.439 \times e^{-0.02352 \times(V+40)} \times\left(\frac{4.5}{K_{o}}\right) \\
\beta_{i}=0.0164 \times e^{0.000942 \times(V+15)} \times\left(\frac{4.5}{K_{o}}\right)^{0.3} \\
\alpha=0.00555 \times e^{0.05547153 \times(V+3)} \\
\beta=0.002357 \times e^{-0.036588 V} \\
\mu=\frac{\alpha_{i} \beta_{2}}{\beta_{i}}
\end{gathered}
$$

A scaling factor ratio of 1.5: 1 in epicardial (EPI) $I_{\mathrm{Ks}}$ to mid-myocardial (MIDDLE) $I_{\mathrm{Ks}}$, was applied as reported in the study of Szabo et al. [14], in order to generate a high, symmetrical T-wave.

\subsection{Drug effect modelling}

Simplified "pore block" [15] was used for drug modelling. The effects of carvedilol on $I_{\mathrm{Kr}}, I_{\mathrm{Ks}}$, were described using Hill coefficient $(\mathrm{nH})$ and half maximal inhibitory concentration $\left(\mathrm{IC}_{50}\right)$ values taken from the literature $\left(10 \mu \mathrm{M}\right.$ carvedilol reduced $I_{\mathrm{Kr}}$ in Wild Typeand $\mathrm{N} 588 \mathrm{~K}-\mathrm{KCNH} 2$ by $92.8 \%$ and $36.0 \%$; it reduced $I_{\mathrm{Ks}}$ by $36.5 \%$ in both conditions) [6].

\subsection{Tissue Simulation}

The mono-domain equation was used to describe the initiation and conduction of APs in the multi-cellular tissue, which is described by the following partial differential equation (PDE):

$$
C_{m} \frac{\partial V_{m}}{\partial t}=-\left(I_{i o n}+I_{s t i m}\right)+\nabla \cdot\left(D \nabla V_{m}\right)
$$

where $D$ is the diffusion coefficient tensor. Equation (23) was solved numerically using a finite-difference PDE solver based on the explicit forward Euler method, as described previously.

We constructed a $15 \mathrm{~mm}$ strand model, and employed a spatial resolution of $0.15 \mathrm{~mm}$. It had 25 nodes for ENDO, 35 nodes for MIDDLE and 40 nodes for EPI cells. $D$ was set at $0.0008 \mathrm{~cm}^{2} / \mathrm{ms}$, which promoted a conduction velocity $(\mathrm{CV})$ of $52 \mathrm{~cm} / \mathrm{s}$.

A pseudo-ECG was calculated by the following expression [16]:

$$
\phi_{e}\left(x^{\prime}\right)=\frac{\alpha^{2}}{4} \int\left(-\nabla V_{m}\right) \cdot \nabla\left(\frac{1}{r}\right) d x
$$

The virtual electrode was placed at a position $2.0 \mathrm{~cm}$ away from the EPI end of the strand.

\section{Results}

Figure 1 shows the simulated cell APs with the modified $I_{\mathrm{Kr}}$ equations. These plots show cell AP $V(\mathrm{mV})$ versus time $t(\mathrm{~ms})$, stimulated by a $1.25 \mathrm{~Hz}$ frequency. Carvedilol prolonged the AP duration in the SQT1 condition.

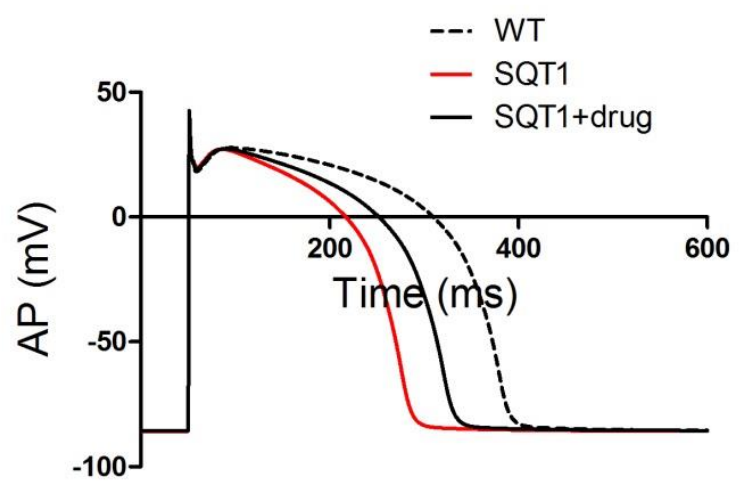

Figure 1. The pharmacological effects of carvedilol on cell action potentials in SQT1 conditions.

A pseudo-ECG was simulated by using a strand model as shown in Figure 2. The QT interval on the ECG was prolonged in SQT1 condition by the application of $10 \mu \mathrm{M}$ carvedilol. It normalized the QT interval in SQT1 from $286 \mathrm{~ms}$ to $364 \mathrm{~ms}$.

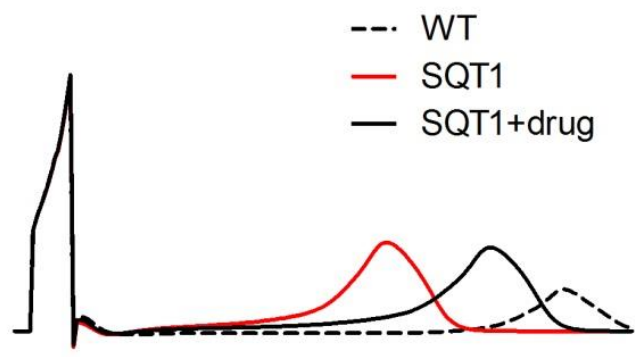

Figure 2. The pharmacological effects of carvedilol on pseudo-ECGs in SQT1 conditions.

\section{Conclusion}

In the present study, we have showed that $10 \mu \mathrm{M} \beta$ adrenoceptor blocker carvedilol produced a therapeutic effect on ventricular electrophysiology in SQT1 conditions, which identified as a potential drug of choice for SQTS treatment. 


\section{Acknowledgements}

This study was supported by the China Scholarship Council (CSC), the National Science Foundation of China (NSFC) under Grants No. 61571165, No. 61572152 and No. 61803318.

\section{References}

1. Luo C, Wang K, Zhang H (2017) Modelling the effects of chloroquine on KCNJ2-linked short QT syndrome. Oncotarget 8: 106511-106526.

2. Luo C, Wang K, Zhang H (2017) In silico assessment of the effects of quinidine, disopyramide and E-4031 on short QT syndrome variant 1 in the human ventricles. PLoS One 12: e0179515.

3. Brugada R, Hong K, Dumaine R, Cordeiro J, Gaita F, et al. (2004) Sudden death associated with short-QT syndrome linked to mutations in HERG. Circulation 109: 30-35.

4. Adeniran I, McPate MJ, Witchel HJ, Hancox JC, Zhang H (2011) Increased vulnerability of human ventricle to re-entrant excitation in hERG-linked variant 1 short QT syndrome. PLoS Comput Biol 7: e1002313.

5. Schimpf R, Wolpert C, Bianchi F, Giustetto C, Gaita F, et al. (2003) Congenital short QT syndrome and implantable cardioverter defibrillator treatment: inherent risk for inappropriate shock delivery. J Cardiovasc Electrophysiol 14: 1273-1277.

6. Bodi I, Franke G, Pantulu ND, Wu K, Perez-Feliz S, et al. (2013) Differential effects of the beta-adrenoceptor blockers carvedilol and metoprolol on SQT1- and SQT2-mutant channels. J Cardiovasc Electrophysiol 24: 1163-1171.

7. Luo C, Wang K, Zhang H (2017) Modeling the effects of amiodarone on short QT syndrome variant 2 in the human ventricles. Conf Proc IEEE Eng Med Biol Soc 2017: 4273-4276.

8. Luo C, Wang K, Zhang H (2017) Effects of islanddistribution of mid-cardiomyocytes on ventricular electrical excitation associated with the KCNQ1linked short QT syndrome. Conf Proc IEEE Eng Med Biol Soc 2017: 3684-3687.

9. Luo C, Wang K, Zhang H (2017) Modelling the effects of quinidine, disopyramide, and E-4031 on short QT syndrome variant 3 in the human ventricles. Physiol Meas 38: 1859-1873.

10. Luo C, Wang K, Zhang H (2017) Effects of amiodarone on short QT syndrome variant 3 in human ventricles: a simulation study. Biomed Eng Online 16: 69.

11. ten Tusscher KH, Panfilov AV (2006) Alternans and spiral breakup in a human ventricular tissue model. Am J Physiol Heart Circ Physiol 291: H1088-1100.

12. ten Tusscher KH, Noble D, Noble PJ, Panfilov AV (2004) A model for human ventricular tissue. Am J Physiol Heart Circ Physiol 286: H1573-1589.

13. O'Hara T, Virag L, Varro A, Rudy Y (2011) Simulation of the undiseased human cardiac ventricular action potential: model formulation and experimental validation. PLoS Comput Biol 7: e1002061.

14. Szabo G, Szentandrassy N, Biro T, Toth BI, Czifra G, et al.
(2005) Asymmetrical distribution of ion channels in canine and human left-ventricular wall: epicardium versus midmyocardium. Pflugers Arch 450: 307-316.

15. Brennan T, Fink M, Rodriguez B (2009) Multiscale modelling of drug-induced effects on cardiac electrophysiological activity. Eur J Pharm Sci 36: 6277.

16. Gima K, Rudy Y (2002) Ionic current basis of electrocardiographic waveforms: a model study. Circ Res 90: 889-896.

Corresponding author:

Cunjin Luo, Ph.D.

E-mail: cunjin.luo@yahoo.co.uk

Mobile Phone: +8618545121660

Address: Key Laboratory of Medical Electrophysiology of Ministry of Education and Medical Electrophysiological Key Laboratory of Sichuan Province, Institute of Cardiovascular Research, Southwest Medical University, Luzhou, Sichuan 646000, China 